\title{
Study of Middle-aged Pulsar Wind Nebulae Showing Large Offsets Between their Pulsar and their VHE Gamma-ray Emission.
}

\section{Tsirou*}

Laboratoire Univers \& Particules de Montpellier (LUPM, CNRS/IN2P3)

E-mail: michelle.tsirou@umontpellier.fr

\section{Y. A. Gallant}

Laboratoire Univers \& Particules de Montpellier (LUPM, CNRS/IN2P3)

E-mail: yves.gallantein2p3.fr

\section{Z. Meliani}

Laboratoire Univers et Théories, (LUTh, Observatoire de Paris, CNRS)

E-mail: zakaria.melianieobspm.fr

\begin{abstract}
Pulsar wind nebulae (PWNe) are clouds of ultra-relativistic highly magnetised particles surrounding rapidly rotating neutron stars. Their emission can range from the radio band up to the very-high-energy (VHE) gamma-ray regime. We focus on the VHE PWN population seen with H.E.S.S. (HESS Collaboration et al., 2018) where many older systems have an observed significant offset of several parsecs between the position of the pulsar and the centroid of VHE emission of the nebula. If due solely to pulsar proper motion such offsets would imply implausible large velocities, above $500 \mathrm{~km} / \mathrm{s}$. We explore, using relativistic (magneto-)hydrodynamic open-source simulations, two possible drivers of such offsets: pulsar proper motion and the inhomogeneity of the medium. We discuss the implications of their physical parameters for the asymmetrical evolution in space of the PWN so as to compare our future results to the observed population of offset VHE middle-aged PWNe.
\end{abstract}

36th International Cosmic Ray Conference -ICRC2019-

July 24th - August 1st, 2019

Madison, WI, U.S.A.

${ }^{*}$ Speaker. 


\section{Evolution of pulsar wind nebulae within composite supernova remnants}

Pulsar wind nebulae (PWNe) are magnetised clouds of ultra-relativistic accelerated positrons and electrons formed around rapidly rotating neutron stars known as pulsars. When the pulsar is young, they are generally found within shell-type supernova remnants (SNRs).

In order to study the morphology of pulsar wind nebulae, it is important to consider both the occurring energy injection that deposit the lepton quota in the PWNe, and the surrounding medium in which they are embedded. In conventional evolution scenarii, the input energy originates from the central pulsar of the system and therefore depends on its characteristics, such as its spin period, defining the rotational energy that in tandem with its magnetic field structure will create a pulsar wind (PW) of cold highly magnetised leptons, and its braking behaviour, which dictates the energy loss rate of the injection. The pulsar wind at its termination shock deposits highly energetic relativistic electrons and positrons that agglomerate in a weakly magnetised zone and form the pulsar wind nebula. The nebula expands by shocking the ambient ejecta matter. The surrounding medium of the PWN is composed by the progenitor star ejecta layered in unshocked and shocked regions. The supernova explosion initially induces a forward blast wave that will propagate outwardly and shock the interstellar medium (ISM). As a result of this forward shock (FS) and the ISM impediment, a reverse shock wave (RS) will travel inwards to the central system, shocking progressively the interior of the remnant, reaching eventually the pulsar wind nebula. Therefore the ambient medium, including the remnant ejecta region and the interstellar medium, will be determined one one hand by the core-collapse supernova energy explosion and ejecta matter bulk and on the other, by the temperature and density structure of the ISM where the supernova happens.

\subsection{Very-high energy gamma-ray pulsar wind nebulae}

Pulsar wind nebulae have been observed from the radio domain, up to the very-high-energy (VHE) gamma-ray energy range thanks to ground-based telescopes and satellites with various resolutions, resulting to a plethora of detected structures. Energy-dependent morphologies for extended sources may bring elements to the understanding of particle transport within these astrophysical objects, such as the tracing of the freshly injected leptons prone to radiation losses, mapped by higher energy radiation, and the site of less energetic thus older particle populations emitting in lower energy bands. In a predominant leptonic process scenario, the synchrotron emission would be associated with the lower energy radiation and the higher energy emission would be a result of inverse Compton scattering on seed photons, thus allowing to compare the extent of the PWN emission in a multi-wavelength frame and possibly prone strong ambient magnetic fields by comparing the gamma-ray morphology to radio and X-ray observations. X-ray radiation and in particular emission above $\mathrm{keV}$ energies may provide a good estimation of the incoming energy budget of the system and the loci of injection.

Sources showing an extended emission and observed with a sufficiently well spatially and energy resolved instrument are excellent sites for system evolution and particle transport investigations, in particular when emission may be correlated to known pulsars and supernova remnants. The High Energy Stereoscopic System (H.E.S.S.) experiment has published a pulsar wind nebulae population study (HESS Collaboration et al. 2018 [1]), based on 14 sources catalogued in the 
H.E.S.S. Galactic Plane Survey (HGPS) [2] which can be firmly associated with known pulsars, along with 5 additional firmly associated sources outside the HGPS catalogue.

\subsection{Significantly offset systems}

H.E.S.S. observations of source morphology based on the HGPS have shown that for a considerable number of pulsar wind nebulae, constituting half of the PWN sample in the published population study [1], there is a significant and measured offset between the VHE gamma-ray emission centroid and the position of the identified associated pulsar. We consider below, based on this study (HESS Collaboration et al. 2018 [1]), the sample of pulsar wind nebulae exhibiting a statistically significant offset, along with the PWN in MSH 15-52 (HESS J1514-591) based on the latest morphological analysis ([3]; H.E.S.S. Collaboration, in prep.). One may notice in Table 1 that the characteristic spin-down age $\tau_{\text {ch }}$ of each system's pulsar is generally greater than $7 \mathrm{kyr}$ which, by considering a conventional spin-down evolution for the pulsar based on the magnetic dipole approximation, may be used as an upper limit to the age of its pulsar wind nebula. This suggests that such offsets may be a typical feature of older VHE-emitting nebulae.

\begin{tabular}{|c|c|c|c|c|c|c|c|c|c|}
\hline $\begin{array}{c}H G P S \\
\text { source name }\end{array}$ & $\begin{array}{l}d_{\text {offset }} \\
\text { (pc) }\end{array}$ & $\begin{array}{c}R_{\mathrm{PWN}} \\
(\mathrm{pc})\end{array}$ & $\begin{array}{c}A T N F \\
\text { pulsar name }\end{array}$ & $\begin{array}{c}D \\
(\mathrm{kpc})\end{array}$ & $\begin{array}{c}\tau_{\mathrm{ch}} \\
(\mathrm{kyr})\end{array}$ & $\begin{array}{c}v_{\mathrm{PM}} \\
\left(\mathrm{km} \cdot \mathrm{s}^{-1}\right)\end{array}$ & $\begin{array}{c}\dot{E}_{\mathrm{sd}} \\
\left(10^{36} \mathrm{erg} \cdot \mathrm{s}^{-1}\right)\end{array}$ & $\begin{array}{c}B_{\text {surf }} \\
\left(10^{12} \mathrm{G}\right)\end{array}$ & $\begin{array}{c}P \\
(\mathrm{~ms})\end{array}$ \\
\hline J1825-137 & $33.0 \pm 6$ & $32.0 \pm 2.0$ & B1823-13 & 3.61 & 21.4 & 399 & 2.8 & 2.8 & 101.5 \\
\hline J1303-631 & $20.5 \pm 1.8$ & $20.6 \pm 1.7$ & J1301-6305 & 10.72 & 11.0 & - & $1 . .7$ & 7.1 & 184.5 \\
\hline J1837-069 & $17.0 \pm 3$ & $41.0 \pm 4.0$ & J1838-0655 & 6.6 & 22.7 & - & 5.6 & 1.9 & 70.5 \\
\hline J1418-609 & $7.3 \pm 1.5$ & $9.4 \pm 0.9$ & J1418-6058 & 1.89 & 10.3 & - & 4.9 & 4.4 & 110.6 \\
\hline J1356-645 & $5.5 \pm 1.4$ & $10.1 \pm 0.9$ & J1357-6429 & 3.1 & 7.3 & - & 3.1 & 7.8 & 166.1 \\
\hline J1420-607 & $5.1 \pm 1.2$ & $7.9 \pm 0.6$ & $\mathrm{~J} 1420-6048$ & 5.63 & 13.0 & - & 10.4 & 2.4 & 68.2 \\
\hline J0835-455 & $2.37 \pm 0.18$ & $2.9 \pm 0.3$ & B0833-45 & 0.28 & 11.3 & 77 & 6.9 & 3.4 & 89.3 \\
\hline J1514-591 & $<4.0$ & $11.1 \pm 2.0$ & B1509-58 & 4.4 & 1.6 & - & 17.5 & 15.4 & 151.3 \\
\hline
\end{tabular}

Table 1: Table of HGPS sources considered as firmly identified pulsar wind nebulae from HESS Collaboration et al. (2018) [1] and characteristics of their embedded pulsar based on the ATNF catalogue [4]. The sorting has been made by increasing offset $d_{\text {offset }}$ between the pulsar position and the peak of VHE gammaray emission. $R_{\mathrm{PWN}}$ is the size of the VHE gamma-ray emission as catalogued in the HGPS based on a multi-Gaussian component model, $D$ is the distance of the pulsar to the observer, $\tau_{\text {ch }}$ is the pulsar characteristic age derived from its measured period and derivative $P$ and $\dot{P}, v_{\mathrm{PM}}$ is the pulsar proper motion (when an available measurement exists in the literature), $\dot{E}_{\mathrm{sd}}$ is the spin-down energy loss rate, and $B_{\text {surf }}$ is the surface magnetic field derived from a dipole configuration.

\section{Plausible drivers behind the observed offsets}

The offset between the position of the pulsar and the fresh lepton injection location has been interpreted as a result of different physical factors. We shall discuss in the following three of the most widely considered drivers behind asymmetrical VHE gamma-ray PWNe morphologies, focusing on their spatial extent related to their pulsar. We consider them as parameters to be varied in our simulation studies.

\subsection{Pulsar initial kick}

In the event of an asymmetrical explosion, supernovae may yield an uneven kick-back of the remaining progenitor core that forms the neutron star and thus convey to the newly born compact 
object a given velocity. The pulsar, having a typical $\sim 1.5 M_{\odot}$ mass in a $\sim 4 \cdot 10^{3} \mathrm{~km}^{3}$ volume shall travel its surroundings within the ballistic limit after the acquired propulsion for a considerable dynamic system evolution age. One plausible cited explanation for the pulsar and VHE emission offset in evolved, "middle-aged" pulsar wind nebulae would be the proper motion of the pulsar, since the pulsar will continuously inject particles into the system but the initial pulsar wind nebula formed at its birth locus will not be replenished and will become a relic. However, as illustrated in Figure 1, in order to explain systematically the observed offset as the traveled distance by the pulsar based on its proper speed, one has to consider a wide range of velocities, differing from the typical measured ranges of $\sim 300 \mathrm{~km} \cdot \mathrm{s}^{-1}$ (Hobbs et al, 2005 [5]). We note that based on the ATNF catalogue-provided proper motion velocities for PSR B1823-13 and PSR B0833-45 shown in Table 1, the first one is not sufficient to explain the reported offset seen by H.E.S.S. for the largely extended pulsar wind nebula J1825-137, by more than a 2.5 factor.

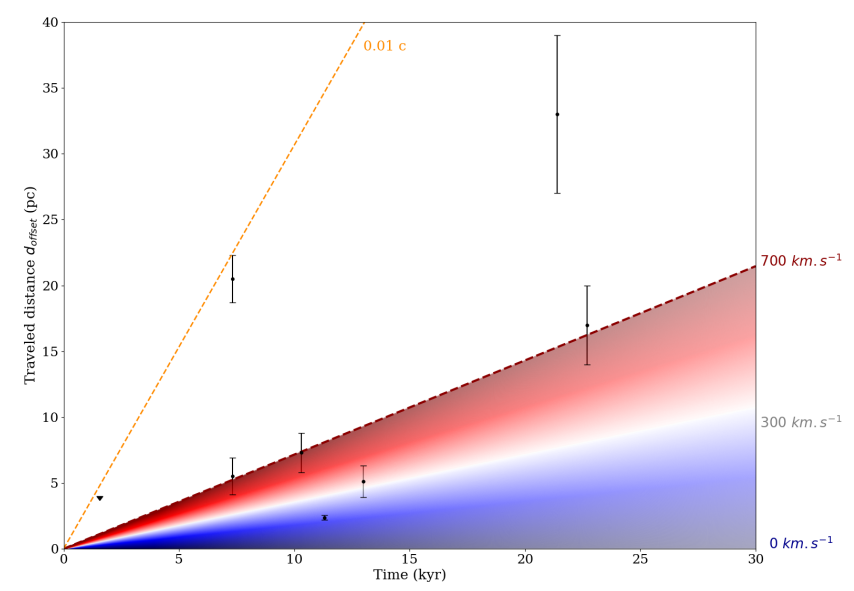

Figure 1: Distance traveled by a kicked pulsar for different ballistic velocities ranging from 0 (dark blue) to 700 (dark red) $\mathrm{km} \cdot \mathrm{s}^{-1}$ and for one percent of the speed of light (orange dashed line). The lighter shaded and white zone between the low (blue) and high (red) velocities delimits the measured typical proper velocity range of pulsars (millisecond pulsars excluded) which is centred around $\sim 250-350 \mathrm{~km} . \mathrm{s}^{-1}$. The measured offsets $d_{\text {offset }}$ of the firmly identified pulsar wind nebulae sample mentioned in Section 1.2 figure for each pulsar spin-down age. We notice that only a few PWNe seem to agree with the expected pulsar proper motion values and that the rest require high velocities $\left(>500 \mathrm{~km} \cdot \mathrm{s}^{-1}\right)$ and for some unrealistic $\sim 10^{3} \mathrm{~km} . \mathrm{s}^{-1}$ to explain the several parsec offset.

Nevertheless, pulsar proper motion is one of the main physical justification evidenced by the overall offset seen for pulsar wind nebulae and PWN candidate sources. We use it as one of the two main parameters to investigate its influence in pulsar-PWN-SNR system evolution with time.

\subsection{Inhomogeneous ambient medium}

Another prime actor behind the offset pulsar wind nebulae populations would be the scale of inhomogeneity of the ambient medium. If the density gradient through the length of the system is strong enough ( 1 to 2 orders of magnitude), the PWN may be tilted by an asymmetric crush caused 
by the shocked ejecta via the reverse shock (RS) propagating towards the centre of the nebula. This will cause a quicker evolution on the higher ambient density immersed side of the nebula, that will no longer be expanding freely in the known as ejecta-dominated stage but will interact with the hot shocked incoming particle flow driven by the RS thus triggering an adiabatic evolution (the SedovTaylor stage) whereas for the lower density surrounded side, its evolution shall continue freely. This will cause a displacement and a strongly asymmetrical evolution.

In our study, we impose a higher density region in the vicinity of the evolving system. We wish to independently study its influence on the intensity and propagation of the reverse shock unevenly crushing the inner nebula and we shall focus in future investigations on the joint effect of pulsar proper motion and of the inhomogeneous ambient medium.

\subsection{Other asymmetry actors}

Another cause for possible pulsar wind nebulae deformation would be an asymmetrical outflow of ions and leptons from the pulsar that would depend on the obliquity angle between the magnetic-field axis emerging from the polar caps of the pulsar and its rotational axis. In our simulations, we compute the energy flux of the pulsar wind based on the assumption that it reaches its maximum in the equatorial direction, thus coupling the Poynting flux to a pulsar wind initial particle flow asymmetry constant (Komissarov and Lyubarsky, 2004 [6]). We do not wish in this study to examine its influence in deforming the termination shock shape oblongly because of the core power computation it would require to consistently spatially resolve the pulsar wind with a great refinement while we simulate the pulsar wind bubble and the supernova remnant blast waves (forward and reverse) in scales greater of 3 to 5 orders. This deformation would render the shape of the PWN elongated along one given axis but would not explain an offset of the position of the pulsar.

Furthermore, asymmetric and irregular shapes of pulsar wind nebulae may be linked to non uniform magnetic-fields. From a phenomenological perspective, there has been evidence for radial, toroidal and non-linear magnetic field topologies which, if strong could shape the PWN and explain to some extent the variety of non-spherical plerions. We do not explore the effect of the magneticfield, as it would require a three-dimensional approach that is beyond the scope of our intended study.

\section{Simulation set-ups}

So as to understand how the proposed explanations cited above, in particular the pulsar proper motion and the heterogeneous interstellar medium, impact the formation of the pulsar wind nebulae at ages of $\sim 10-20 \mathrm{kyr}$ and to quantify each physical factor onto the asymmetric evolution, we use the open-source available MPI-AMRVAC 2.0 software (MPI-Adaptive Mesh Refinement Versatile Advective Code)[8] using a relativistic magneto-hydrodynamic physics module implemented to the latest version based on previous work of Meliani et al. (2012)[7]. In our paper in preparation, we shall compare our 2D results with available published studies with non-relativistic hydrodynamic approaches (e.g. Temim et al. 2015 [9], Slane et al. 2018 [10], and references therein). 


\subsection{Adaptive mesh-refinement parallel magneto-hydrodynamic code}

In this proceeding we present one-dimensional (1D) relativistic hydrodynamic simulations that we used as a consistency check for both numerical and initial physical set-ups by cross-examining them with previous studies such as the simulations of van der Swaluw et al. (2001) [11] and Del Zanna et al. (2004) [12].

We solve numerically the relativistic version of Euler's equations of hydrodynamics (we set the magnetisation and thus the magnetic field to zero) in a 1D spherical geometry, with CGS units, for matter density $\rho$, total energy density $\varepsilon$ and momentum density $\rho v$. We use a second order predictor-corrector type 'twostep' scheme for time integration, a Minmod flux limiter and HLL flux scheme. We set, using the inherit tree nature of the code, a total of 96 cells in blocks with each of an 8 cell dimension for a grid with a maximum radius of $6 \cdot 10^{19} \mathrm{~cm}$. Each refinement ratio between levels is by default set to 2 and we proceed by adapting the regions of each system component (located via appointed tracers) by an adequate interval of allowed refinement levels based on the order of physical variables' local small-scale fluctuations. We opt for this method to make sure that, 1) the pulsar wind and its termination shock are sufficiently resolved in comparison with the rest of the system so that the density and pressure of the relativistic wind is bounding the inside of the PWN without being rendered into too coarse a block by the adaptive nature of the refinement, and 2) the reverse and forward shock waves are resolved as well but not too finely so that the Rayleigh-Taylor instabilities at the contact discontinuity between the shocked ejecta and ISM do not dominate the small-scale fluctuations. We define for the pulsar wind and nebula region a minimum of 5 and maximum of 11 levels of refinement, and for the supernova remnant shock region an interval from 1 to 8 refinement levels.

\subsection{One-dimensional relativistic hydrodynamic framework}

We present in Figure 2 our 1D hydrodynamic simulations that we used for computational time conservation purposes to check the evolution of the composite SNR - PWN systems based on our different sets of initial parameters.

We simulated a supernova by depositing an initial explosion energy of $E_{\mathrm{SN}}=10^{51} \mathrm{erg}$ in $5 M_{\odot}$ of uniform-density ejecta, and imposing a radial velocity profile with a maximum ejecta velocity of $5700 \mathrm{~km} \cdot \mathrm{s}^{-1}$, ensuring that $\sim 97 \%$ of $E_{\mathrm{SN}}$ is in the form of kinetic energy. The initially set ejecta radius corresponds to an evolution time of $80 \mathrm{yr}$. The pulsar, characterised by a typical spin-down braking index $n=3$ and an initial spin-down energy loss rate $\dot{E}_{0}=510^{38} \mathrm{erg} . \mathrm{s}^{-1}$, is being switched on $20 \mathrm{yr}$ after the start of the simulation and powers a relativistic wind (of Lorentz factor $\gamma=10$ ) with an initially input termination shock radius of $\sim 10^{15} \mathrm{~cm}$ and pulsar wind nebula size of $\sim 10^{17}$ $\mathrm{cm}$ at the moment of the switch. We let the system evolve in a homogeneous simulated interstellar medium with density $n_{0}=1.0 \mathrm{~cm}^{-3}$ density and temperature $T=10^{4} \mathrm{~K}$.

We find a compatible evolution of the pulsar wind nebula size with van der Swaluw et. al, 2001 [11], but with a slightly different normalisation which is likely due to the different initial set-up.

\subsection{Towards a two-dimensional relativistic (magneto-)hydrodynamic frame}

We are currently working on simulations in a two-dimension cylindrical framework $(r, z, \theta)$ where the system is let to evolve radially with an imposed proper motion in the $z$ axis. We do not 

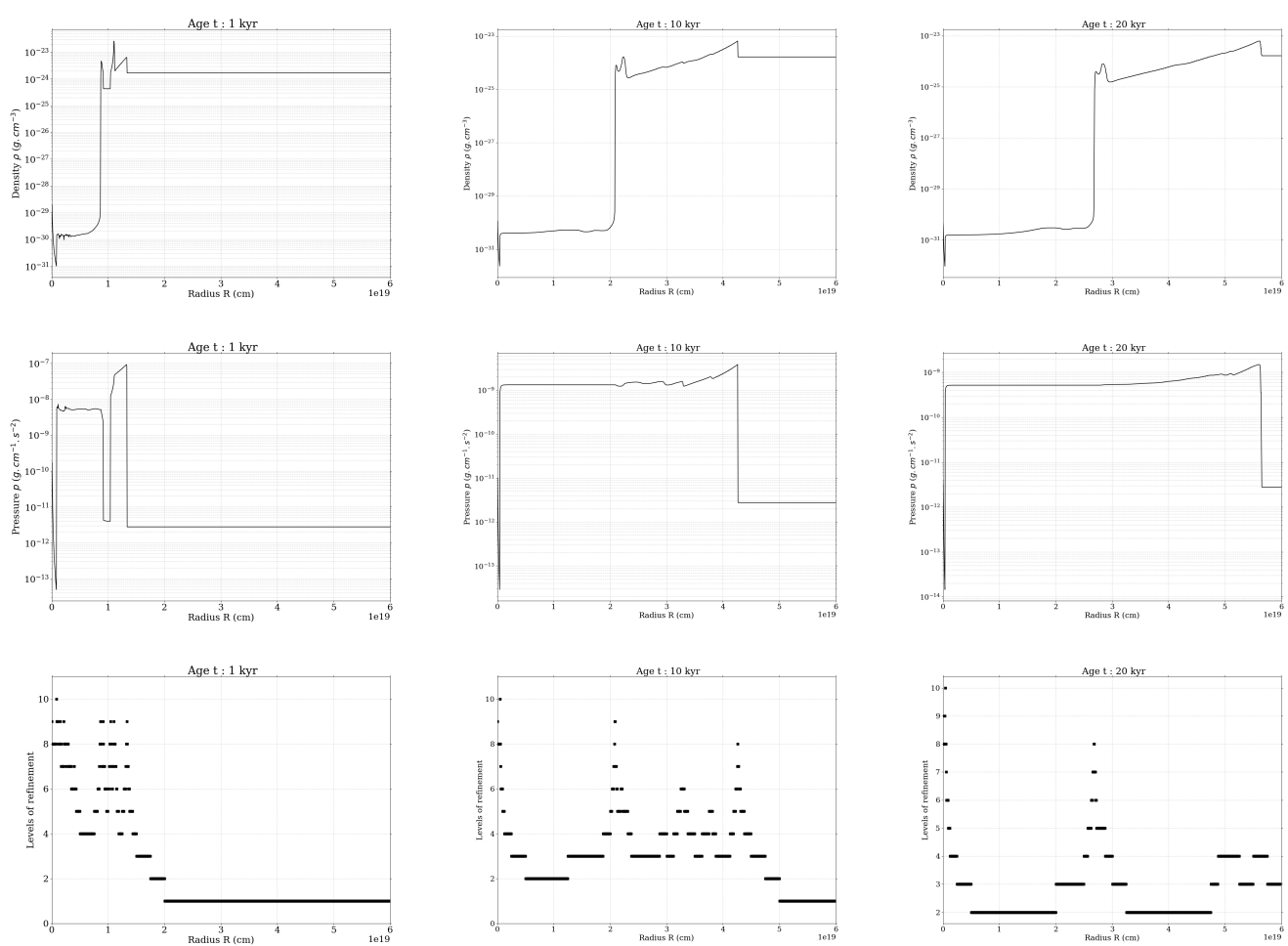

Figure 2: Radial profiles of density, pressure and refinement level from top to bottom, for simulated ages of 1000,10000 and 20000 years in a relativistic $(\gamma=10)$ hydrodynamic frame. The evolution of the termination shock, pulsar wind nebula and the supernova remnant's forward and reverse shock waves are in agreement with published non-relativistic [11] and relativistic [12] studies, taking into account the differences in the simulation configuration set-ups.

present them in this proceeding because of their very preliminary nature but report them in Tsirou, Meliani and Gallant (2019, in prep).

We simulate the proper motion of the pulsar by setting it static in our simulation frame and by setting both the interstellar medium and the supernova remnant in motion. Thus, at the early stages of the simulation run, we switch on the pulsar in a moving $z$-axis frame, which is numerically easier to handle than affecting the proper motion into a non-ab initio set object.

We as well separated the grid into two density media, one significantly denser than the other, to simulate the differed and delayed evolution of the joint supernova remnant - embedded pulsar wind nebula system. In future work, we will impose a gradient of density rather than the currently strong contrasted media so as to investigate a more realistic ambient medium.

Motivated by the large scale offsets between the position of the pulsar and centroid of the very-high-energy gamma-ray emission of several pulsar wind nebulae, we have begun a study with relativistic hydrodynamic and magneto-hydrodynamic simulations. We aim to characterise the possible lengths of offsets expected with high pulsar proper velocities and compare them to the ones caused by ambient medium inhomogeneities so as to understand if these observed offsets are a typical feature in the evolution of pulsar wind nebulae. 


\section{References}

[1] H.E.S.S. Collaboration, et al., 2018, A\&A, 612, A2 [astro-ph.HE/1702.08280]

[2] H.E.S.S. Collaboration, et al., 2018, A\&A, 612, A1 [astro-ph.HE/1804.02432]

[3] M. Tsirou, Y. Gallant, R. Zanin \& R. Terrier, for the H.E.S.S. Collaboration, 2017, International Cosmic Ray Conference, 301, 681 [astro-ph.HE/1709.01422]

[4] R.N. Manchester, G.B. Hobbs, A. Teoh, A. \& M. Hobbs, 2005, Astron. J., 129, 1993-2006 [astro-ph.HE/0412641]

[5] G. Hobbs, D.R. Lorimer, A.G. Lyne \& M. Kramer, 2005, MNRAS, 360, 974

[6] S.S. Komissarov \& Y.E. Lyubarsky, 2004, MNRAS 349, 779-792

[7] R. Keppens, Z. Meliani, A. J. van Marle, et al., 2012, Journal of Computational Physics, 231, 718 [astro-ph.SR/1710.06140]

[8] C. Xia, J. Teunissen, I. El Mellah, et al., 2018, ApJS, 234, 30 [astro-ph.SR/1710.06140]

[9] T. Temim, P. Slane, C. Kolb, et al., 2015, ApJ, 808, 100 [astro-ph.HE/1506.03069]

[10] P. Slane, I. Lovchinsky, C. Kolb, S.L. Snowden, T. Temim, J. Blondin, et al., 2018, ApJ, 865, 86

[11] E. van der Swaluw, A. Achterberg, Y.A. Gallant \& G. Tóth, 2001, A\&A, 380, 309

[12] L. del Zanna, E. Amato, N. Bucciantini, 2004, A\&A, 421, 1063-1073 [astro-ph.HE/0404355] 